\title{
Automatic Recognition of Facial Expressions in Image Sequences: A Review
}

\author{
R A Patil \\ MNIT Jaipur \\ rapatil_rtg@yahoo.co.in
}

\author{
Vineet Sahula \\ MNIT Jaipur \\ sahula@ieee.org
}

\author{
A. S. Mandal \\ CEERI Pilani \\ atanendusekhar.mandal@gmail.com
}

\begin{abstract}
For human beings, facial expression is one of the most powerful and natural way to communicate their emotions and intensions. A human being can detect facial expressions without effort, but for a machine it is very difficult. Automatic facial expression recognition is an interesting and challenging problem. Automatic facial expression recognition systems can be mainly used for human computer interaction and data driven animation. There are three sub problems while designing automatic facial expression recognition system, face detection, extraction of the facial expression information, and classification of the expression. A system that performs these operations more accurately and in real time would be crucial to achieve a humanlike interaction between man and machine. This paper reviews the past work done in solving these problems for image sequences.
\end{abstract}

\section{INTRODUCTION}

Machine vision has been defined as the automatic acquisition and analysis of images to obtain desired data for interpreting a scene or controlling an activity. For a machine vision system, it is not necessary to copy all the details of the human visual system, but it is important to understand the true complexity behind its power and flexibility. Current machine vision research concerns, not only understanding the process of vision, but also designing effective vision systems for various real world applications. Facial expression recognition system is an example of machine vision system.

For a human being the most powerful, and natural way of communicating their emotions and intensions is facial expression. A human being can detect facial expressions in a scene without effort. But, to develop an automatic system that performs this task is rather difficult. A demand of automatically extracting facial expression information has been continuously increasing. Automatic facial expression analysis is an interesting and challenging problem, and impacts important applications in many areas such as human computer interaction, and data driven animation. Though much progress has been made, recognizing facial expression with a high accuracy remains challenge due to subtlety, complexity and variability of facial expressions. There are six prototypical (or basic) facial expressions, i.e. surprise, fear, sadness, anger, disgust and happiness according to the previous researches, which are known to be universal across human ethnicities and cultures.

The goal of this paper is to survey the work done in automating facial expression analysis in facial image sequences. Section II describes three basic problems related to facial expression analysis. These problems are face detection in a facial image sequence, facial expression data extraction and facial expression classification. Here, we discuss few systems which deal with each of these problems. We conclude in section III

\section{FACIAL EXPRESSION ANALYSIS}

Facial expression recognition problem can be divided into three sub problems. (1) face detection (2) feature extraction (3) classification. To detect the face from the image sequence is first and the very important step. Next step is to develop mechanism for extracting the facial features from the observed facial image sequence. The facial features are the prominent features of the various parts of the face- eyebrows, eyes, nose, mouth, and chin. The feature extraction step is often referred to as tracking the face and its features, in the scene. The final step is to develop a classifier, which will classify a facial expression into one of the basic facial expressions [1].

\section{A. Face detection}

Most of the methods of facial expression recognition assumes that the conditions under which a facial image sequence is obtained, are controlled. Usually, the image sequence has the face in frontal view. Hence, the presence of a face in the scene is ensured and some global location of the face in the scene is known a priori. However, determining the exact location 
of the face in a digitized facial image is a more complex problem. The scale and the orientation of the face can vary from image to image. The presence of noise and occlusion makes the problem even more complex [1].

By casual inspection of a scene, human being detect faces without effort, under bad illumination or from a long distance. If a face is partially occluded, human being can perceive a whole face, because perceptual system of human being fills in the missing parts. To do this by a machine is very difficult. An ideal system should be capable of dealing with wide range of head movements, from frontal view to profile view. It should be able to handle changes in lighting conditions as well as distractions like glasses, and facial hair like moustache, beard.

Lajevardi and Lech [2] have proposed a method in which the face area of an image was detected using the Viola-Jones method based on the Haar-like features and AdaBoost learning algorithm. The Viola and Jones method [3] is an object detection algorithm providing competitive object detection rates in real-time. It was mainly designed for the face detection. The features are derived from pixels selected from rectangular area, which is imposed over the picture and which shows high sensitivity to the vertical and horizontal lines. AdaBoost [4] is an adaptive learning algorithm that can be used in conjunction with many other learning algorithms to improve their performance.

Cohn [5], Wang [6], Black \& Yacoob [7], and Kotsia [8] have not mentioned the technique that they used for face detection. Summary of the surveyed methods for automatic face detection is presented in Table I. Yacoob and Davis [9] assume that rectangles enclosing the facial features have been initially placed before the onset of tracking. Essa and Pentland [10] use the eigenspace method of Pentland et al. to locate faces. They applied Principal Component Analysis (PCA) on a sample of 128 facial images to create eigenfaces. The eigenfaces define the subspace of sample images, i.e., socalled "face space". To detect the presence of faces in an image sequence, a spatio-temporal filtering is performed, the filtered image is thresholded in order to analyze "motion blobs", and each motion blob that can represent a human head is then evaluated as a single image [1].

Kimura and Yachida [11] utilize a Potential Net for face representation. By using the centers of the eyes and the center of the mouth an input image is normalized first. This algorithm applies an integral projection method, which synthesizes the color and the edge information. Then, the Potential Net is fitted
Table I

SUMMARY OF THE METHODS FOR AUTOMATIC FACE DETECTION

\begin{tabular}{|l|l|l|}
\hline Reference & Method & Remarks / Limitations \\
\hline \hline Lajevardi[2] & Viola-Jones method & $\begin{array}{l}\text { No rigid head rotations } \\
\text { allowed }\end{array}$ \\
\hline Essa [10] & Eigen space method & $\begin{array}{l}\text { rigid head motions } \\
\text { allowed in horizontal } \\
\text { direction }\end{array}$ \\
\hline Kimura [11] & Potential net fitting & $\begin{array}{l}\text { No rigid head rotations } \\
\text { allowed }\end{array}$ \\
\hline
\end{tabular}

to the normalized image to model the face and its movement. The face should be without facial hair and glasses and in a direct face-to-face position with the camera [1].

\section{B. Facial feature Extraction}

Once the face is detected from the image sequence, the next step is to extract the information about the shown facial expression. Because of high variability in the types of faces, it is very difficult for the machine to extract facial features. Variations in lighting conditions, head movements, non frontal views, various distractions like glasses, facial hair makes the problem more difficult. An ideal system should be able to recognise all visually distinguishable facial expressions. Well defined technique of feature extraction is prerequisite for achieving this [1].

Lajevardi and Lech [2] use a holistic approach, which extracts features from a picture of the whole face. The features were generated using the log-Gabor filters. The log-Gabor filters can be constructed with an arbitrary bandwidth that can be optimized to produce a filter with minimal spatial extent. The log-Gabor filters have Gaussian transfer functions when viewed on the logarithmic frequency scale. It was therefore postulated that the log-Gabor functions having extended tails at the high frequency ends should be able to encode natural images more efficiently by providing a good representation of the high frequency components. Five scales and eight orientations were implemented to extract features from face images. This lead to 40 filter transfer functions $\{\mathrm{H} 1, \mathrm{H} 2$, ...,H40 representing different scales and orientations. The objective was to choose only the log-Gabor filters which minimize the absolute value of the spectral difference between the original image filter output and the noisy image filter output. Initially, the error was calculated for all of the training images, and for each image a set of 4 log-Gabor filters with 
the smallest value of the spectral difference was selected. The selected optimal subset of 4 log-Gabor filters allowed reducing the features dimension from 40 to only 4 arrays of size $60 \times 60$ for each image. The feature arrays were transferred to feature vectors of length 3600 for each image.

Yacoob and Davis [9] approach is independent of tracking individual point motions. It is based on a statistical characterization of the motion patterns in specified regions of the face. They develope a region tracker for rectangles enclosing the face features. Each rectangle encloses one feature of interest, so the flow computation within the region is not "contaminated" by the motions of other facial features. To simplify the modeling of the eyebrows, they define the rectangles to include the eyes, and then subtract the rectangle of the eye from the combined rectangle. The tracking algorithm integrates spatial and temporal information at each frame. In order to enhance the tracking the statistics of the motion directions within a rectangle are used to verify translation of rectangles upward and downward and verify scaling of the rectangles. With the help of universal expression descriptions proposed by Ekman and Friesen, and motion patterns of expression proposed by Bassili, they prepare dictionary of facial feature actions (motion based feature description of facial actions). The dictionary is divided into components, basic actions of these components, and motion cues. The components are defined qualitatively and relative to the rectangles surrounding the face regions. Using component's visible deformations, the basic actions are determined. Using optical flow within these regions, the basic actions are determined.

Essa and Pentland [10] applied the eigenspace method to automatically track the face in the scene and extract the positions of the eyes, nose, and mouth. The method employs eigenfeatures approximated using PCA on a sample of 128 images. To detect the location of the prominent facial features in a given image, the distance of each feature-image from the relevant feature space is computed using a FFT and a local energy computation. The extracted position of the prominent facial features is further used to normalize the input image. A 2D spatiotemporal motion energy representation of facial motion estimated from two consecutive normalized frames is used as a dynamic face model. Essa and Pentland use the optical flow computation method proposed by Simoncelli who uses a multiscale coarse-to-fine Kalman filter to obtain motion estimates and error-covariance information. It computes first a mean velocity vector, which represents the estimated flow from consecutive normalized facial images of a video sequence. The flow covariances between different frames are stored and used together with the recursive continuous time Kalman filter to calculate the error predictions, based on previous data, and to obtain a corrected, noise-free $2 \mathrm{D}$ motion field. The method has been applied to frontal-view facial image sequences [1].

Kimura and Yachida [11] made the use of a Potential Net for face representation. First they compute the edge image by applying a differential filter, to fit the Potential Net to a normalized facial image. Then the image is filtered using a Gaussian filter, in order to extract the external force, which is a smooth gradient of the edge image. The filtered image is called "potential field" to which the elastic net model is placed. The net deforms further governed by the elastic force of the potential field. They fit a Potential Net to each frame of the examined facial image sequence. Then they compare pattern of the deformed net with the pattern extracted from an expressionless face (neutral face normally the first frame of a sequence) and the variation in the position of the net nodes is used for further processing. They built an emotion space by applying PCA on six image sequences of three expressions anger, happiness, and surprise shown by a single person gradually, from expressionless to a maximum. The eigenspace spanned by the first three principal components has been used as the emotion space, onto which an input image is projected for a quantified emotional classification [1]. The method is used only for frontal faces. The face should be without facial hair and glasses.

Cohn et al. [5] utilize a model of facial landmark points localized around the facial features. The points are marked with hand in the first frame of an examined image sequence. A hierarchical optical flow method is used to track the optical flows of $13 \times 13$ windows surrounding the landmark points in the rest of the frames. The displacement of each landmark point is calculated by subtracting its normalized position in the first frame from its current normalized position (all frames of an input sequence are manually normalized). The displacement vectors, calculated between the initial and the peak frame, represent the facial information used for recognition of the displayed facial actions [1].

Wang et al. [6] proposed a method in which he utilize 19 facial feature points (FFPs) - seven FFPs to preserve the local topology and 12 FFPs for facial expression recognition. The FFPs are treated as nodes of a labeled graph that are interconnected with links representing the Euclidean distance 
between the nodes. It is assumed that we know the initial location of the FFPs in the first frame of an input image sequence. To track the FFPs in the rest of the frames, he use a system that consists of two layers, a memory layer and an input layer. The correspondence between the FFPs tracked in two consecutive frames is treated as a labeled graph matching problem where the antecedent frame is treated as the memory layer and the current frame as the input layer. The degree of expression change is determined based on the displacement of the FFPs in the consecutive frames [1].

Black and Yacoob [7] made the use of local parametrized models of image motion for facial expression recognition. To derive the midlevel predicates that describe the motion of the facial features, they made use of motion parameters (e.g., translation and divergence). Each midlevel predicate is represented in a form of a rule, where the left part of the rule is a comparison of a motion parameter to a certain threshold and the right part of the rule is the derived predicate. The thresholds are dependent on the face size in the image and were set empirically from a few sequences. They did not give a full list of the midlevel predicates and the number of different facial actions that the method can recognize is not known. For each of six basic emotional expressions, they developed a model represented by a set of rules for detecting the beginning and ending of the expression. The rules are applied to the predicates of the midlevel representation [1].

kotsia and Pitas [8] proposed a method which is based on mapping and tracking the facial model Candide onto the video frames. The proposed method is semi-automatic. The user has to manually place candide wire frame model at the first frame of the image sequence under examination. The tracking system allows candide wire frame to follow the the facial expression over time till it reaches its highest intensity. The candide grid is deformed at each video frame. A subset of the Candide grid nodes is chosen, that predominantly contribute to the formation of the facial deformations described by the FACS. A well known Kanade Lucas Tomasi tracker is used for tracking facial features in subsequent frames. The geometrical displacement of candide grid nodes, the difference of each node coordinates at the first and the last frame of the facial image sequence, is used as an input to a SVMs classifier.

Summary of the surveyed methods for automatic facial feature extraction is presented in Table III.
Table II

COMPARING THE METHODS FOR FACIAL EXPRESSION DATA EXTRACTION

\begin{tabular}{|c|c|c|}
\hline Reference & Method & Remarks / Limitations \\
\hline $\begin{array}{l}\text { Lajevardi } \\
\text { and Lech[2] }\end{array}$ & $\begin{array}{l}\text { Log Gabor filters with } \\
\text { gaussian transfer } \\
\text { functions }\end{array}$ & $\begin{array}{l}\text { Only front view faces } \\
\text { without hair and glasses } \\
\text { allowed }\end{array}$ \\
\hline $\begin{array}{l}\text { Yacoob and } \\
\text { Davis [9] }\end{array}$ & $\begin{array}{l}\text { statistical } \\
\text { characterization of } \\
\text { motion pattern in } \\
\text { specified regions of face }\end{array}$ & $\begin{array}{l}\text { Only front view faces } \\
\text { without hair and glasses } \\
\text { allowed }\end{array}$ \\
\hline $\begin{array}{l}\text { Essa and } \\
\text { Pentland } \\
{[10]}\end{array}$ & Optical flow method & $\begin{array}{l}\text { Front views, Face without } \\
\text { hair and glasses, light } \\
\text { variation allowed }\end{array}$ \\
\hline $\begin{array}{l}\text { kimura and } \\
\text { Yachinda } \\
{[11]}\end{array}$ & $\begin{array}{l}\text { Potential net fitting to } \\
\text { normalized face image } \\
\text { by Gaussian filter }\end{array}$ & $\begin{array}{l}\text { Only front view faces } \\
\text { without hair and glasses } \\
\text { allowed }\end{array}$ \\
\hline $\begin{array}{l}\text { Cohn et. al } \\
{[5]}\end{array}$ & $\begin{array}{l}\text { Optical flow algorithm } \\
\text { of Lucas Kanade }\end{array}$ & $\begin{array}{l}\text { Front views, Face without } \\
\text { hair and glasses, manual } \\
\text { labeling on first frame }\end{array}$ \\
\hline $\begin{array}{l}\text { Wang et. al } \\
{[6]}\end{array}$ & labeled graph fitting & $\begin{array}{l}\text { Front views, Face without } \\
\text { hair and glasses, manual } \\
\text { labeling on first frame }\end{array}$ \\
\hline $\begin{array}{l}\text { Black and } \\
\text { Yacoob [7] }\end{array}$ & $\begin{array}{l}\text { Local parametrized } \\
\text { model of image motion, } \\
\text { optical algorithm }\end{array}$ & $\begin{array}{l}\text { head motion and light } \\
\text { variation allowed }\end{array}$ \\
\hline $\begin{array}{l}\text { Kotsia and } \\
\text { Pitas[8] }\end{array}$ & $\begin{array}{l}\text { Candide wire frame } \\
\text { model fitting and } \\
\text { Pyramidal Kanade Lucas } \\
\text { Tomasi tracker }\end{array}$ & $\begin{array}{l}\text { Frontal views, face } \\
\text { without glass allowed } \\
\text { Manual fitting of model } \\
\text { on first frame is necessay }\end{array}$ \\
\hline
\end{tabular}

\section{Facial Expression Classification}

Finally we have to classify the extracted facial expression information into a particular facial action or basic emotion. The techniques used for classification by surveyed systems are templated based, neural network, rule based, and support vector machines (SVM).

In a template based approach the encountered facial expression is compared to the templates defined for each expression category. The best match decides the class of the shown facial expression. It is impossible to model different facial actions with a finite set of templates, as there are infinite number of combinations of different facial actions and their intensities [1].

In neural network based approach, a facial expression is classified according to the categorization process that the network learned during a training phase.

In rule based classification, the facial expression is classified into the basic emotion categories based on previously encoded facial actions. The prototypic expressions, which characterize 
the emotion categories, are first described in terms of facial actions. Then the shown expression is compared to the prototypic expressions defined for each of the emotion categories and classified in the optimal fitting category [1]

In SVM approach, a hyperplane is constructed between different classes during training phase. The algorithm then classifies the encountered facial expression into one of the class during test phase.

Cohn et al. [5] utilize template based method. Separate group variance-covariance matrices were used for classification. He used image sequences (504) containing 872 facial actions displayed by 100 subjects. He used two discriminant functions for three facial actions of the eyebrow region, two discriminant functions for three facial actions of the eye region and five discriminant functions for the nine facial actions of the nose and mouth region. The accuracy was $92 \%$ for the eyebrow region, $88 \%$ for the eye region, and $83 \%$ for the nose/mouth region.

Essa and Pentland [10] generated the spatio temporal templates for six different expressions. Each template has been delimited by averaging the patterns of motion generated by two subjects showing a certain expression. The euclidean distance between the motion energy template and the observed image motion energy is used for measuring similarity or dissimilarity. He tested it on 52 frontal view image sequences of 8 people showing six different expressions with accuracy rate of $98 \%$.

Kimura and Yachida [11] built an emotion space by applying PCA on six image sequences of three expressions anger, happy, and surprise shown by a single person gradually from neutral to maximum. The eigenspace spanned by the first three principal components has been used as the emotion space, onto which an input image is projected for a quantified emotional classification [1].

Wang et. al [6] utilize 19 points labeled graph, out of these 12 points are used for expression recognition. For each of three emotion categories anger, happy, and surprise they use 12 average FFPs B spline curves, one for each FFPs, to construct the expression model. Each expression model has been defined from 10 image sequences displayed by 5 subjects. The category of expression is decided by determining the minimal distance between the actual trajectories of FFPs and the trajectories defined by the models. The degree of expression change is determined based on the displacements of the FFPs in the consecutive frames. He tested it on 29 image sequences of 3 emotional expressions shown by 8 subjects.
The average recognition rate was $95 \%$ [1].

Lajevardi and Lech [2] utilize log Gabor filter for feature extraction. These features are classified using Naive Bayesian Classifier (NB). He assumes that the presence of a particular feature of a class is unrelated to the presence of any other feature. The classification decision is made using the following formula:

$$
C=\operatorname{argmax}\left\{P\left(C_{i}\right) \prod P\left(f_{i} / C_{i}\right)\right\}
$$

where $C_{i}=1, \ldots \ldots, 6$ is class label and $f_{i}$ is feature related to each sample.

Yacoob and Davis [9] designed a rule based system that combines some expression descriptions. He prepared rules in identifying the onsets of the begining and the ending of each facial expression e.g. for Anger, beginning is inward lowering brows and mouth compaction and ending is outward raising brows and mouth expansion. These rules are applied to the mid level representation to create a complete temporal map describing the evolving facial expression.

Black and Yacoob [7], utilize local parametrized models of image motion to represent rigid head motions and nonrigid facial motions within the local facial areas. The motion parameters (e.g., translation and divergence) are used to derive the midlevel predicates that describe the motion of the facial features. Each midlevel predicate is represented in a form of a rule, where the left part of the rule is a comparison of a motion parameter to a certain threshold and the right part of the rule is the derived predicate. For each of six basic emotional expressions, they developed a model represented by a set of rules for detecting the beginning and ending of the expression. The rules are applied to the predicates of the midlevel representation. The method has been tested on 70 image sequences containing 145 expressions shown by 40 subjects ranged in ethnicity and age. The expressions were displayed one at the time. The achieved recognition rate was 88 percent [1].

Kotsia and Pitas [8] utilize multiclass SVM for expression classification. The geometrical displacements i.e. the grid deformation feature vector is used as input to a multiclass SVM. He considered six classes for the experiments, each one representing one of the basic facial expressions (anger, disgust, fear, happy, sad, surprise). The SVM system classifies the set of grid deformation vector into one of the six class. He used 
Table III

COMPARING THE METHODS FOR FACIAL EXPRESSION CLASSIFICATION

\begin{tabular}{|c|c|c|c|}
\hline Ref. & Method & Test sequences & Accuracy \\
\hline \begin{tabular}{l|} 
Lajevardi \\
Lech \\
{$[2]$}
\end{tabular} & $\begin{array}{l}\text { Naive Bayesian } \\
\text { Classifier }\end{array}$ & $\begin{array}{l}388 \text { sequences of } 100 \\
\text { subjects from Cohn } \\
\text { Kanade database }\end{array}$ & $68.9 \%$ \\
\hline $\begin{array}{l}\text { Yacoob } \\
\text { Davis[9] }\end{array}$ & $\begin{array}{l}\text { rule based, prepared } \\
\text { dictionary of rules }\end{array}$ & $\begin{array}{l}46 \text { sequences of } 32 \\
\text { subjects }\end{array}$ & \\
\hline $\begin{array}{l}\text { Essa } \\
{[10]}\end{array}$ & $\begin{array}{l}\text { Spatio temporal motion } \\
\text { energy templates }\end{array}$ & $\begin{array}{l}22 \text { sequences of } 8 \\
\text { subjects }\end{array}$ & $100 \%$ \\
\hline $\begin{array}{l}\text { kimura } \\
{[11]}\end{array}$ & 3D emotion space (PCA) & & \\
\hline $\begin{array}{l}\text { Cohn } \\
{[5]}\end{array}$ & Discriminant functions & $\begin{array}{l}504 \text { sequences of } 100 \\
\text { subjects }\end{array}$ & $88 \%$ \\
\hline $\begin{array}{l}\text { Wang } \\
{[6]}\end{array}$ & $\begin{array}{l}\text { Averaged Bsplines of } \\
\text { feature trajectories }\end{array}$ & $\begin{array}{l}29 \text { sequences of } 8 \\
\text { subjects }\end{array}$ & $95 \%$ \\
\hline $\begin{array}{l}\text { Black } \\
{[7]}\end{array}$ & $\begin{array}{l}\text { temporal consistency of } \\
\text { the mid level predicates } \\
\text { which describes the } \\
\text { motion of the facial } \\
\text { features }\end{array}$ & $\begin{array}{l}70 \text { sequences of } 40 \\
\text { subjects }\end{array}$ & $88 \%$ \\
\hline $\begin{array}{l}\text { Kotsia } \\
{[8]}\end{array}$ & Multi class SVM & $\begin{array}{l}\text { sequences from Cohn } \\
\text { Kanade database }\end{array}$ & $91.4 \%$ \\
\hline
\end{tabular}

Cohn Kanade database for training and testing.

Summary of the methods used for facial expression classification is given in Table IV.

\section{CONCLUSION}

Automatic recognition of facial expressions by a machine is still a challenging problem. The challenge is due to high variability in the types of faces. The problem is divided into three sub problems face detection, feature extraction and facial expression classification. Most of the existing systems assume that the presence of the face in a scene is ensured. Most of the systems deal with only feature extraction and classification, assuming that face is already detected. In addition, assumptions are also made like (i) images are frontal view, (ii) illumination is constant, (iii) light source is fixed, (iv)face has no facial hair and glasses, (v) the subjects are young without wrinkles, and (vi) subjects are immovable. It is unrealistic to expect these assumptions in application domains of human behavior interpretation and human computer interface. Almost all the systems classify facial expressions into one of the six basic emotion-categories proposed by Ekman and Friesen. Every time it is not possible that all facial expressions, able to be displayed on the face, can be classified under the six basic emotion-categories.

Only one of the surveyed method propsed by Essa and
Pentland allows rigid head motions and deal with facial images with hair and eyeglasses. Another point which none of the systems are focused is "filling in" missing parts of the observed face and reading a whole face even when a part of it is occluded. In a system developed by Kotsia and Pitas only geometrical information is required for SVM classifier, no texture information is required. But for tracking they used optical flow computation, where illumination is an important factor. The system can not be used for colored and non frontal facial images.

If facial expression recognition systems are to be operated autonomously, current feature extraction methods have to be improved, and manual adjustments during initialization should be made automatic. The system should be able to recognize facial expressions from non frontal views with hair and glasses allowing head rotations also. It should be able to handle occlusions also, and any expression irrespective of six basic facial expressions should be recognized.

\section{REFERENCES}

[1] M. Pantic and L. J. M. Rothkrantz, "Automatic analysis of facial expressions: The state of the art," IEEE Transactions on Pattern analysis and machine intelligence, vol. 22, pp. 1424-1445, Dec. 2000.

[2] S. Lajevardi and M. Lech, "Facial expression recognition from image sequences using optimized feature selection," Image and Vision Computing New Zealand, 2008. IVCNZ 2008. 23rd International Conference, pp. 1-6, 2008.

[3] P. Viola and M. Jones, "Robust real-time object detection," Cambridge Research Laboratory Technical report series, pp. 1-30, February 2001.

[4] P. Viola and M. Jones, "Rapid object detection using a boosted cascade of simple features," Computer Vision and Pattern Recognition, 2001. CVPR 2001. Proceedings of the 2001 IEEE Computer Society Conference on, pp. 511-518, February 2001.

[5] J. F. Cohn, A. J. Ziochower, J. J. lien, and T. kanade, "Feature point tracking by optical flow discriminates subtle differences in facial expression," Proc. Int'l Conf. Automatic Face and Gesture Recognition, pp. 396-401, 1998.

[6] M. Wang, Y. Iwai, and M. Yachindai, "Expression recognition from timesequential facial images by use of expression change model," Proc. Int'l Conf. Automatic Face and Gesture Recognition, pp. 324-329, 1998.

[7] M. J. Black and Y. Yacoob, "Recognizing facial expressions in image sequences using local parameterized models of image motion," lnt'l J. Computer Vision, vol. 25, no. 1, pp. 23-48, 1997.

[8] I. Kotsia and I. Pitas, "Facial expression recognition in image sequences using geometric deformation features and support vector machines," IEEE Transactions on Image Processing, vol. 16, pp. 172-187, Jan. 2007.

[9] Y. Yacoob and L. Davis, "Recognizing human facial expressions from long image sequences using optical flow," IEEE Transactions on Pattern Analysis and Machine Intelligence, vol. 18, pp. 636-642, June 1996.

[10] I. A. Essa and A. P. Pentland, "Coding, analysis, interpretation, and recognition of facial expressionsl," IEEE Trans. Pattern Analysis Machine Intelligence, vol. 19, pp. 757-763, July 1997.

[11] S. Kimura and M. Yachida, "Facial expression recognition and its degree estimation," Proc. Computer Vision and Pattern Recognition, pp. 295300, 1997. 